\title{
Coexisting massive and massless Dirac fermions in symmetry-broken bilayer graphene
}

Keun Su Kim ${ }^{1,2}$, Andrew L. Walter ${ }^{3}$, Luca Moreschini ${ }^{1}$, Thomas Seyller ${ }^{4}$, Karsten Horn ${ }^{2}$, Eli Rotenberg ${ }^{1}$ and Aaron Bostwick ${ }^{1 \star}$

${ }^{1}$ Advanced Light Source, E. O. Lawrence Berkeley National Laboratory, Berkeley, CA 94720, USA, ${ }^{2}$ Department of Molecular Physics, Fritz-Haber-Institut der Max-Planck-Gesellschaft, Faradayweg 4-6, 14195 Berlin, Germany, ${ }^{3}$ Donostia International Physics Centre, Manuel Lardizábal 4, E-20018 San Sebastián, Spain, ${ }^{4}$ Lehrstuhl für Technische Physik, Universität Erlangen-Nürnberg, Erwin-Rommel-Strasse 1, 91058 Erlangen, Germany.

^e-mail: abostwick@|bl.gov.

Charge carriers in bilayer graphene are widely believed to be massive Dirac fermions ${ }^{1-3}$ that have a bandgap tunable by a transverse electric field ${ }^{3,4}$. However, a full transport gap, despite its importance for device applications, has not been clearly observed in gated bilayer graphene ${ }^{5-7}$, a long-standing puzzle. Moreover, the low-energy electronic structure of bilayer graphene is widely held to be unstable towards symmetry breaking either by structural distortions, such as twist $^{8-10}$, strain ${ }^{11,12}$, or electronic interactions $s^{7,13,14}$ that can lead to various ground states. Which effect dominates the physics at low energies is hotly debated. Here we show both by direct band-structure measurements and by calculations that a native imperfection of bilayer graphene, a distribution of twists whose size is as small as $\sim 0.1^{\circ}$, is sufficient to generate a completely new electronic spectrum consisting of massive and massless Dirac fermions. The latter is robust against strong electric fields, and has a novel topology in momentum space consisting of closed arcs having an exotic chiral pseudospin texture, which can be tuned by varying the charge density. The discovery of this unusual Dirac spectrum not only complements the framework of massive Dirac fermions, widely relevant to charge transport in bilayer graphene, but also supports the possibility of valley Hall transport $^{15}$.

Symmetry breaking and its effect on electronic structure is an enduring topic in the study of graphene. Graphene has a conical spectrum with a point crossing at the Dirac energy $\left(E_{\mathrm{D}}\right)$, which is protected by the symmetry of constituent sublattices (indexed $A$ and $B$ ), and robust against applied electric fields ${ }^{1}$. Bilayer graphene (BG), stacked in the Bernal sequence ( $A B$-stacking or $A B-B G$ ) is characterized by a massive Dirac spectrum with two pairs of parabolic bands ${ }^{1-4}$. An external electric field breaks layer symmetry, resulting in a bandgap at $E_{\mathrm{D}}$ (Fig. 1a $)^{3,4}$. In principle, a massless Dirac spectrum could appear in $B G$ if the two layers were exactly aligned (AA-stacking or $A A-B G)^{16,17}$. The resulting bandstructure consists of two Dirac cones merged into a "Dirac circle" at $E_{\mathrm{D}}$ 
(Fig. 1b). However, this interesting structure is energetically unfavourable compared to Bernal stacking ${ }^{16,17}$.

Angle-resolved photoemission spectroscopy (ARPES) is an essential tool, which can not only directly probe electronic states, but also give key information on their symmetry. Using ARPES, we show that practically obtainable BG samples consist of large symmetry-broken regions, whose layers are minutely rotated (twisted) relative to each other. Consequently, large regions of AA-like stacking with a slight interlayer twist (tAA$B G$ ) are naturally generated. At large energy, $t A A-B G$ has a similar spectrum to $A A-B G$, having extra Dirac crossings, which we verify directly by ARPES. At low energy, however, the combination of twist and applied field breaks interlayer-coupling and potential symmetry, leading to a new band topology (Fig. 1c) with unmistakable and surprisingly strong signatures in ARPES data. Our results demonstrate the presence of strong rotational symmetry breaking in the electronic structure, arising from twist angles whose presence cannot be easily ruled out in fabricated devices. Even in the absence of external fields, detectable rotational symmetry breaking will arise from such small twist angles, and this can affect transport properties of gated BG devices.

Figure $1 \mathrm{~d}$ shows an ARPES spectrum of quasi-freestanding $B G^{18-20}$, taken at the $\mathrm{K}$ point along $k_{\mathrm{x}}$ (inset of Fig. 1i), so that only one branch of the spectrum is visible ${ }^{21,22}$. The samples are $p$-doped with $E_{\mathrm{D}}$ about $0.15 \mathrm{eV}$ above the Fermi energy $\left(E_{\mathrm{F}}\right)$, similar to quasi-freestanding monolayer graphene ${ }^{18,19}$. The substrate induces a weak potential difference $U$ between the two layers, and a small bandgap opens at $E_{\mathrm{D}}{ }^{3,4}$, above $E_{\mathrm{F}}$. As expected for AB-BG, there are two parabolic $\pi$ bands, consistent with tight-binding band calculations $^{3}$ (black lines overlaid). However, there is another, linear band $(C 1$, red arrow) that starts from the lower $\pi$ band and crosses $E_{\mathrm{F}}$.

We deposited potassium, which induces electron-doping and increases the bandgap at $E_{\mathrm{D}}{ }^{4}$. Figure 1e,f shows two doping levels with corresponding band calculations (black lines). The $C 1$ band first reaches $E_{F}$ in the bandgap of AB-BG (Fig. 1e), and then merges with the lower $\pi^{\star}$ band (Fig. 1f). At this doping, another linear band (C2, blue arrows) appears, starting from the upper $\pi$ band and merging with the upper $\pi^{*}$ band through the bandgap of $A B-B G$. Thus, even when $E_{F}$ is within the bandgap of $A B-B G$ in an electric field (Fig. 1e), the system is not fully insulating due to two new metallic bands.

The origin of these bands is revealed by the photon-energy dependence of their intensity, which, while nominally constant in monolayer graphene, strongly oscillates in $B G$ due to interlayer interference (similar to Young's double slits) ${ }^{21,22}$. The oscillation period is determined by the number of layers and their separation, regardless of stacking order $^{21}$. Our data for C1 and C2 clearly show out-of-phase oscillatory behaviour with the period of BG (Supplementary Figs. S1 and S2), confirming their bilayer origin. That the bands move rigidly with doping makes many-body origins ${ }^{19}$ unlikely.

These new bands are also identified in highly $n$-doped spectra along $k_{y}$ (Fig. 2a), where bands of both positive and negative slope have nominally equal intensity ${ }^{22}$. There are two $X$-shaped crossings at $E_{\mathrm{D} 1}$ and $E_{\mathrm{D} 2}$, and two nearly vertical features near $E_{\mathrm{D}}$, which are not expected for AB-BG (black lines in Fig. 2b). These features are equivalent 
to those in Fig. 1 by reflection across the $\mathrm{K}$ point, and suggest the existence of two conical bands separated in energy (blue and red lines in Fig. 2b). Supporting this picture, constant-energy maps at $E_{\mathrm{D} 1}$ and $E_{\mathrm{D} 2}$ show a clear point crossing (Fig. 2d,e), and the map at $E_{\mathrm{D}}$ shows a circular feature (Fig. 2h).

This circular feature near $E_{D}$ arises from the overlap of states of opposite chirality (Fig. 2b), a hallmark of AA-type stacking. This is confirmed by its photon-energy dependence. In general, a single Dirac cone has vanishing intensity towards either $\pm k_{\mathrm{x}}$ (as in Fig. $2 \mathrm{~d}, \mathrm{e}$ ) for a given pseudospin chirality ${ }^{21,22}$, independent of photon energy for our measurement conditions $^{23}$. In contrast, our data at $E_{\mathrm{D}}$ (Fig. $2 \mathrm{~h}-\mathrm{j}$ ) show a clear intensity reversal with photon energy (red and blue arrows). This is possible only if the circular feature has contributions from both chiralities (Supplementary Fig. S3). Thus, the observed spectrum consists of two parts, one from $A B-B G$, which opens a bandgap at $E_{D}$ under external electric fields (Fig. 1a) ${ }^{4}$, and the other with linear bands at $E_{\mathrm{D}}$ as expected for AA-BG (Fig. $1 \mathrm{~b}$ or $1 \mathrm{c})^{16,17}$.

Spectral simulations including finite broadening and matrix-element effects are shown in Figs. 1g-i and 2c, which successfully reproduce the experimental data only if AA-BG is included (full dataset in Supplementary Fig. S2). The best fit yields the interlayercoupling energies of AA-BG between the same $\left(\gamma_{1}=-0.22 \pm 0.02 \mathrm{eV}\right)$ and opposite sublattices $\left(\gamma_{3}=-0.03 \pm 0.01 \mathrm{eV}\right)$, and the velocity of Dirac fermions $\left(c^{\star} \approx 1.1 \times 10^{6} \mathrm{~m} / \mathrm{s}\right)$. Furthermore, the area enclosed by the circular feature at $E_{\mathrm{D}}$ (Fig. $2 \mathrm{~h}$ ) is predicted to systematically increase in size with applied field ${ }^{17}$. We demonstrate this in Fig. $2 \mathrm{k}$, where we show that the area of the circular feature is widely tunable, by an order of magnitude. This is a unique characteristic of the massless Dirac spectrum in AA-BG, as compared to the fixed zero density at $E_{\mathrm{D}}$ in monolayer graphene ${ }^{1}$.

We now show that the AA-stacked graphene must be twisted to account for a crucial symmetry breaking observed in our data. Figure $2 \mathrm{~d}-\mathrm{h}$ shows a series of $k$-maps taken simultaneously near $E_{\mathrm{D}}$. The symmetry of intensity patterns at $E_{\mathrm{D}}$ (Fig. $2 \mathrm{~h}$ ), where only AA-BG features are present, is quite distinct from those at higher and lower energies (Fig. 2d-g), which are symmetrical with respect to $k_{y}$, imposed by lattice-mirror symmetry $^{22}$. Unlike these patterns that have maximum intensity along $k_{\mathrm{x}}$ (vertical dotted lines in Fig. $2 \mathrm{~d}-\mathrm{g}$ ), the data in Fig. $2 \mathrm{~h}$ have a maximum along the $45^{\circ}$-rotated dotted line. This feature was consistently observed with different photon energy (Fig. $2 \mathrm{~h}-\mathrm{j}$ ) and measurement geometry (the linear polarization of photons along $k_{\mathrm{x}}$ or $k_{\mathrm{y}}$ ), confirming its intrinsic origin. No such spectral asymmetry is expected for normal AA-BG (or any other graphene systems), unless its lattice symmetry is broken.

A scenario for this symmetry breaking, energetically more favourable than perfect $A A-$ $B G$, is a minute twist that produces large and sequential domains of $A B-, A A-$, and $B A-$ stacked $B G^{8-10}$. Recent atomic-scale microscopic studies have observed such domains surprisingly often ${ }^{24-26}$, and we have also found spectroscopic signatures of twists, whose angle $\theta$ varies randomly in between $0.1 \sim 0.4^{\circ}$ (Supplementary Fig. S4). Additionally, rotational disorder on the order of $\pm 0.15^{\circ}$ was recently reported in monolayer graphene ${ }^{27}$. Such distortions are in a subtle disorder regime; for example, only one atomic misfit in 
$100 \times 100 \mathrm{~nm}^{2} \mathrm{BG}$ yields $\theta \approx 0.14^{\circ}$. These twists are an order of magnitude smaller than the out-of-plane roughness $\left(\theta \sim 2^{\circ}\right)$ observed in suspended $\mathrm{BG}^{28}$, and can be easily generated by far-flung wrinkles, ripples, or closed edges ${ }^{24-26,28}$. These are well-known and universal structural features in graphene materials due to strong lateral $\sigma$ bonds. The cohesive energies of $A A-B G$ and $A B-B G$ differ by only a few meV per atom ${ }^{17}$, in contrast to the cost of creating an atomic defect (several eV per atom). Therefore, in response to a local stress, BG would rather induce a twist involving thousands of atoms, rather than create an atomic-scale defect. The effect of this long-range relaxation, however, is often disregarded in interpreting mesoscopic experimental data.

A small twist leads to electronic domain separation due to the limited coherence length of quasi-particles (few tens $\mathrm{nm}$ ) with respect to the typical size of stacking domains $\left(140 \times 140 \mathrm{~nm}^{2}\right.$ for $\left.\theta \sim 0.1^{\circ}\right)$. The local electronic structure of such domains can reasonably be approximated with a simple tight binding model based on a recent theory ${ }^{10}$, which we extended for small twist angles and external fields (see Methods). Figure $3 a$ shows the result for $\theta=0.15^{\circ}$ and $U=0.4 \mathrm{eV}$. The overall dispersions are unchanged from $\mathrm{AA}-\mathrm{BG}$, but small gaps are opened at band crossings $\left(E_{\mathrm{D} 1}, E_{\mathrm{D} 2}, E_{\mathrm{D}}\right)$ and those near $E_{\mathrm{D}}$ occur, not at the same energy, but in a plane tilted with respect to $k_{\mathrm{x}}$ and $k_{\mathrm{y}}$. While the gaps are not directly observable in our data due to finite lifetime and resolution, their presence induces a modulation of the constant-energy map at $E_{\mathrm{D}}$, resulting in rotated spectral symmetry (Fig. 3b,c), just as observed experimentally (Fig. $2 \mathrm{~h}, \mathrm{i}$ ). The identical map simulated without the matrix-element effect (Fig. 3e) confirms that this rotated symmetry comes from the band topology itself. In our calculations, both overall dispersions and rotated symmetry at $E_{\mathrm{D}}$ are not sensitive to the specific $\theta$ value $(\theta$ $<0.5^{\circ}$ ) or even to its direction $( \pm \theta)$ (Fig. 3e,f and Supplementary Fig. S5 and Movie). This explains the well-defined spectral features in our data, even though the twists might have a distribution of signs and angles.

This twist induces a small splitting of the Dirac point $\Delta k$. This splitting, together with interlayer-potential asymmetry $U$ and interlayer-coupling parameter $\gamma_{3}$, results in a unique low-energy topology near $E_{\mathrm{D}}$, shown in Fig. 4a for two representative twist angles. The bands have small gaps at Dirac crossings (Fig. 4b), which are asymmetrically placed with respect to both $k_{\mathrm{x}}$ and $k_{\mathrm{y}}$. The central energy $E_{0}$ and magnitude $\Delta$ of the gap strongly vary with azimuthal angle $\phi(\mathrm{Fig} .4 \mathrm{c}, \mathrm{d})$. The gap is maximum along green arrows (anti-nodal direction), and vanishes towards purple arrows, leaving only two Dirac nodes (nodal direction). This nodal and anti-nodal gap symmetry is the physical origin of rotated spectral symmetry observed in Fig. 3.

For small $\theta$, the band topology is predominantly determined by symmetry-breaking terms $\left(\gamma_{3}\right.$ and $U$ ), which are constant for non-zero $\theta$, rather than the K-point splitting $\Delta k$, which is proportional to $\theta$. This makes it nearly independent of the value and sign of $\theta$. Instead, it has inversely rotated symmetry for $-U$ or $\gamma_{1} \gamma_{3}<0$ (Fig. $3 g$ ). As the sign of $U$ is known for our sample ${ }^{4}$, it follows that $\gamma_{1}$ and $\gamma_{3}$ have the same sign. We also considered the possibilities of $\operatorname{strain}^{11}$ and interlayer shear ${ }^{12}$, but could not reproduce the key aspects of our data. 
This band topology has an unusual zero-energy surface at $E_{\mathrm{D}}$ (Fig. 4e). It is not a Dirac circle as expected for untwisted AA-BG, but rather two closed arcs, one of electrons and the other of holes. For $0<\theta \lesssim 0.3^{\circ}$, these two arcs face each other at $\pm k_{\mathrm{x}}$, and have opposite chirality. At greater angles, there is an electronic topological transition (Lifshitz transition) $^{10}$, whereupon the arcs are oriented along $\pm k_{\mathrm{y}}$ (the maximum in the spectral function remains at $\sim 45^{\circ}$ ). This is a unique pseudospin texture, which can be viewed as a pseudospin analog of the Rashba effect. This Dirac-arc topology may be exploited in new quantum transport phenomena such as the valley Hall effect ${ }^{15}$. For twists larger than $0.5^{\circ}$, new features appear in the bandstructure that we can rule out from the data (Supplementary Fig. S5).

Our results show that even tiny imperfections of BG can dramatically change its fundamental Dirac spectrum. The resultant band structure is the superposition of a massive and a new, previously unobserved massless Dirac spectrum of BG, as illustrated in Fig. 1a,c. This band superposition and the absence of moire bands ${ }^{8,9}$ can be explained by the lack of long-range structural and electronic coherence in real-space, as limited by the typical grain size (few hundreds $n m$ ). The signature of tAA-BG is present in all of our samples, and could also be identified in previously reported data on the same ${ }^{29}$ and related ${ }^{4}$ systems. This is natural, given that such small twists are almost inevitable in practical samples. Thus, this work should have wide relevance to describing charge carriers in $B G$ and solving puzzling issues such as subgap conductance via variable-range hopping ${ }^{5}$, the absence of reduced velocity ${ }^{8-10,30}$, and the role of manybody interactions in broken-symmetry states ${ }^{7,14}$, including nematic-like phases ${ }^{13}$.

\section{Methods}

Sample preparation. Samples were fabricated with semiconducting $6 \mathrm{H}-\mathrm{SiC}(0001)$ wafers with a dopant concentration of $1 \times 10^{18} \mathrm{~cm}^{-3}$. These wafers were first etched in hydrogen to remove the surface polishing damage, and thermally annealed in a flow of argon to grow two graphene layers (including the buffer-layer) on the surface ${ }^{20}$. The number of grown graphene layers was controlled by annealing time and temperature, and checked by $\mathrm{x}$-ray photoemission spectroscopy. After that, the sample was annealed at $850^{\circ} \mathrm{C}$ in a flow of hydrogen to terminate residual bonds of the buffer-layer with the substrate, resulting in quasi-freestanding bilayer graphene on the surface ${ }^{18-20}$. This intercalation method produces samples with a larger grain size and a better uniformity than the conventional one formed by vacuum-annealing ${ }^{4}$. The samples prepared in this way were transferred through the air to ARPES apparatus, and briefly annealed up to $500{ }^{\circ} \mathrm{C}$ to clean the surface in a ultrahigh vacuum. We scanned over the samples with a high-flux and focused photon beam ( $\sim 50 \mu \mathrm{m}$ in diameter) to find the best spots where no signature of monolayer or trilayer is detected in ARPES spectra. The quality of our samples and their thickness were further confirmed by sharp ARPES spectra and photon-energy scans as shown in Fig. 1 and Supplementary Fig. S1.

ARPES experiments. Experiments were conducted at two different endstations in the Advanced Light Source, at beamline 7.01 (most data) and at beamline 4.01 (data in Supplementary Fig. S1), equipped with Scienta R4000 and R8000 analyzers (VG-Scienta, Sweden), respectively. Data 
were collected at $10-15 \mathrm{~K}$ with the photon energy of $45-125 \mathrm{eV}$. Energy and momentum resolutions were better than $30 \mathrm{meV}$ and $0.01 \AA^{-1}$, and the base pressure was $3-5 \times 10^{-11}$ torr. Potassium deposition was done in-situ by a commercial (SAES) getter source.

Tight-binding band calculations. We employed the standard $4 \times 4$ tight-binding Hamiltonian ${ }^{3,16}$ for the low-energy band structure of bilayer graphene with $U$. The key difference between the AB$B G$ and $A A-B G$ models is interlayer-coupling terms, which can be generally written in $2 \times 2$ offdiagonal submatrix as,

$$
T=\left(\begin{array}{ll}
c_{\mathrm{AA}} & c_{\mathrm{AB}} \\
c_{\mathrm{BA}} & c_{\mathrm{BB}}
\end{array}\right)
$$

where $c_{i j}$ is the coupling between sublattices $i$ and $j$ in different layers. For the AB-BG model ${ }^{22}$, $c_{\mathrm{AB}}=\gamma_{1}, c_{\mathrm{BA}}=\gamma_{3} f(k)$, and $c_{\mathrm{AA}}, c_{\mathrm{BB}}=\gamma_{4} f(k)$, where $f(k) \approx-\frac{\sqrt{3} a}{2}\left(k_{x}-i k_{y}\right)+\frac{a^{2}}{8}\left(k_{x}+i k_{y}\right)^{2}$, and $a$ is the lattice constant. The coupling parameters are $\gamma_{1}=-0.35 \sim-0.46 \mathrm{eV}$ (depending on $n$, as discussed in ref. 4), $\gamma_{3}=-0.30 \mathrm{eV}$, and $\gamma_{4}=-0.04 \mathrm{eV}$. For the AA-BG model ${ }^{16}, c_{\mathrm{AA}}, c_{\mathrm{BB}}=\gamma_{1}$ and $c_{\mathrm{AB}}, c_{\mathrm{BA}}=\gamma_{3} f(k)$. These coupling parameters are quantified from the experimental data as $\gamma_{1}=$ $-0.22 \mathrm{eV}$ and $\gamma_{3}=-0.03 \mathrm{eV}$. The $U$ value is estimated from the AB-BG bands, as described in ref. 4 , and the same value is applied for AA-BG or tAA-BG.

Our tight-binding model for twisted bilayer graphene was inspired by a recent theory by Mele ${ }^{10}$. Unlike the typical continuum model ${ }^{8,9}$ based on infinite twist with specific $\theta$, the moiré effect is not included in our model for a distribution of minute twists. This is because a long moire periodicity for $\theta<0.5^{\circ}$ cannot be long-range ordered, as limited by the typical grain size (few hundreds $\mathrm{nm}$ ), and the distribution of $\pm \theta$ would substantially limit their correlation length. The limited correlation length of moiré potentials makes corresponding Fourier components in $k$-space negligible, leaving only coherent coupling between two separated Dirac points with respect to the $\mathrm{K}$ point. This is supported by the absence of any long-range periodicity in ARPES, whose intensity is proportional to the Fourier amplitude of given potentials. In this case, the typical $12 \times 12$ Hamiltonian of twist ${ }^{8}$ is reducible to a simple $4 \times 4$ matrix, similar to those for $A B-B G$ and $A A-B G$. There are two major twist-induced differences, (1) the splitting of Dirac points $(\Delta k)$ accompanying the pseudospinphase shift, and (2) constant interlayer-coupling parameters in $T$ (dropping $f(k)$ term above) ${ }^{10}$. Since these coupling parameters are related to the local atomic configuration, we adopted for tAA-BG the experimentally determined values from $\mathrm{AA}-\mathrm{BG}\left(c_{\mathrm{AA}}, c_{\mathrm{BB}}=\gamma_{1}\right.$ and $\left.c_{\mathrm{AB}}, c_{\mathrm{BA}}=\gamma_{3}\right)$, which is close to "complementary state" $\left(c_{\mathrm{AA}}, c_{\mathrm{BB}} \gg c_{\mathrm{AB}}, c_{\mathrm{BA}}\right)$ in ref. 10.

Spectral simulations. Our model is based on the standard spectral-function formula in a Lorenzian form, and the sublattice-interference effect as,

$$
I(k, E, h v) \propto \frac{\sigma(E)}{\left(E-E_{b}(k)\right)^{2}+\sigma(E)^{2}} \cdot f_{\mathrm{FD}}(E) \cdot M(k, E, h v)
$$

where $E_{\mathrm{b}}(k)$ is the band dispersion, $\sigma(E)$ is the spectral width, $f_{\mathrm{FD}}(E)$ is the Fermi-Dirac function, and $M(k, E, h v)$ is the interference-related matrix element, depending on the photon energy $(h v)$. $E_{\mathrm{b}}$ is taken from tight-binding bands. $\sigma(E)$ is set by energy resolution, $30-50 \mathrm{meV}$, at $E_{\mathrm{F}}$, from which it monotonically increases with binding energy. This increment of the spectral width with binding energy is to take into account the self-energy effect. $E_{\mathrm{F}}$ is determined by $n$ of each experimental 
data. $M(k, E, h v)$ of bilayer graphene is calculated according to ref. 22 , which considers interlayer and intralayer interference of sublattices, and intensity attenuation for the photoelectron-escape depth. The $h v$ dependence of $M(k, E, h v)$ calculated with the interlayer separation of $\sim 3 \AA^{-1}$ is shown in Supplementary Fig. $S 1$. For $M(k, E, h v)$ calculations for $A B-B G$, we assumed the equal population of two inequivalent configurations of $A B-B G$ and $B A-B G$.

\section{References}

1. Geim, A. K. \& Novoselov, K. S. The rise of graphene. Nature Mater. 6, 183-191 (2007).

2. Novoselov, K. S. et al. Unconventional quantum Hall effect and Berry's phase of $2 \pi$ in bilayer graphene. Nature Phys. 2, 177-180 (2006).

3. McCann, E. \& Fal'ko, V. I. Landau-level degeneracy and quantum Hall effect in a graphite bilayer. Phys. Rev. B 96, 086805 (2006).

4. Ohta, T., Bostwick, A., Seyller, T., Horn, K. \& Rotenberg, E. Controlling the electronic structure of bilayer graphene. Science 313, 951-954 (2006).

5. Oostinga, J. B., Heersche, H., B., Liu, X., Morpurgo, A. F. \& Vandersypen, L. M. K. Gate induced insulating state in bilayer graphene devices. Nature Mater. 7, 151-156 (2008).

6. Li, J., Martin, I., Büttiker, M. \& Morpurgo, A. F. Topological origin of subgap conductance in insulating bilayer graphene. Nature Phys. 7, 38-42 (2010).

7. Weitz, R. T., Allen, M. T., Feldman, B. E., Martin, J. \& Yacoby, A. Broken-symmetry states in doubly gated suspended bilayer graphene. Science 330, 812-816 (2010).

8. Lopes dos Santos, J. M. B., Peres, N. M. R. \& Castro Neto, A. H. Graphene bilayer with a twist: Electronic structure. Phys. Rev. Lett. 99, 256802 (2007).

9. Bistritzer, R. \& MacDonald, A. H. Moiré bands in twisted double-layer graphene. Proc. Natl Acad. Sci. USA 108, 12233-12237 (2011).

10. Mele, E. J. Band symmetries and singularities in twisted multilayer graphene, Phys. Rev. $B$ 84, 235439 (2011).

11. Mucha-Kruczyński, M., Aleiner, I. L. \& Fal'ko, V. I. Strained bilayer graphene: Band structure topology and Landau level spectrum. Phys. Rev. B 84, 041404 (2011).

12. Son, Y. -W., Choi, S. -M., Hong, Y. P., Woo, S. \& Jhi, S. -H. Electronic topological transition in sliding bilayer graphene. Phys. Rev. B 84, 155410 (2011).

13. Mayorov, A. S. et al. Interaction-driven spectrum reconstruction in bilayer graphene. Science 333, 860-863 (2011).

14. Velasco Jr, J. et al. Transport spectroscopy of symmetry-broken insulating states in bilayer graphene. Nature Nanotech. 7, 156-160 (2012).

15. Xiao, D., Yao, W. \& Niu, Q. Valley-contrasting physics in graphene: Magnetic moment and topological transport. Phys. Rev. Lett. 99, 236809 (2007).

16. Ho, J. H., Lu, C. L., Hwang, C. C., Chang, C. P. \& Lin, M. F. Coulomb excitations in AA- and AB-stacked bilayer graphites. Phys. Rev. B 74, 085406 (2006).

17. Nanda, B. R. K. \& Satpathy, S. Strain and electronic field modulation of the electronic structure of bilayer graphene. Phys. Rev. B 80, 165430 (2009).

18. Riedl, C., Coletti, C., Iwasaki, T., Zakharov, A. A. \& Starke, U. Quasi-free-standing epitaxial graphene on SiC obtained by hydrogen intercalation. Phys. Rev. Lett. 103, 246804 (2009).

19. Bostwick, A. et al. Observation of plasmarons in quasi-freestanding doped graphene. Science 328, 999-1002 (2010).

20. Speck, F. et al. The quasi-free-standing nature of graphene on $\mathrm{H}$-saturated $\mathrm{SiC}(0001)$. Appl. Rev. Lett. 99, 122106 (2011). 
21. Ohta, T. et al. Interlayer interaction and electronic screening in multilayer graphene investigated with angle-resolved photoemission spectroscopy. Phys. Rev. Lett. 98, 206802 (2007).

22. Mucha-Kruczyński, M. et al. Characterization of graphene through anisotropy of constantenergy maps in angle-resolved photoemission. Phys. Rev. B 77, 195403 (2008).

23. Gierz, I., Henk, J., Höchst, H., Ast, C. R. \& Kern, K. Illuminating the dark corridor in graphene: Polarization dependence of angle-resolved photoemission spectroscopy on graphene. Phys. Rev. B 83, 121408 (2011).

24. Lauffer, P. et al. Atomic and electronic structure of few-layer graphene on SiC(0001) studied with scanning tunneling microscopy and spectroscopy. Phys. Rev. B 77, 155426 (2008).

25. Liu, Z., Suenaga, K., Harris, P. J. F. \& lijima, S. Open and closed edges of graphene layers. Phys. Rev. Lett. 102, 015501 (2009).

26. Borysiuk, J., Soltys, J. \& Piechota, J. Stacking sequence dependence of graphene layers on $\mathrm{SiC}(0001)$-Experimental and theoretical investigation. J. Appl. Phys. 109, 093523 (2011).

27. Walter, A. L. et al. Small scale rotational disorder observed in epitaxial graphene on SiC(0001). New J. Phys. 15, 023019 (2013).

28. Meyer, J. C., Geim, A. K., Katsnelson, M. I., Novoselov, K. S., Booth T. J. \& Roth, S. The structure of suspended graphene sheets. Nature 446, 60-63 (2007).

29. Watcharinyanon, S. et al. Hydrogen intercalation of graphene grown on $6 \mathrm{H}-\mathrm{SiC}(0001)$. Surf. Sci. 605, 1662-1668 (2011).

30. Berger, C. et al. Electronic confinement and coherence in patterned epitaxial graphene. Science 312, 1191-1196 (2006).

Correspondence and requests for materials should be addressed to Aaron Bostwick.

\section{Acknowledgements}

This work and ALS were supported by the U.S. Department of Energy, Office of Sciences under Contract No. DE-AC02-05CH11231. K.S.K. acknowledges support by NRF Grant funded by the Korean Government (NRF-2011-357-C00022). A.L.W. acknowledges support by the Max Planck Society. L.M. acknowledges support by Grant PA00P2-136420 from the Swiss National Science Foundation (SNSF). Work in Erlangen was supported by the DFG in the framework of the Priority Program 1459 'Graphene'. We thank F. Speck, M. Ostler, and F. Fromm for assistance during sample preparation, and J. Denlinger for assistance during measurement.

\section{Author contributions}

E.R., K.H. and A.B. conceived the experiments. K.S.K. performed the experiments, data analysis, and interpretations with help of L.M. and supervision of A.B. and E.R. A.L.W. and T.S. fabricated the samples, and performed the preliminary experiments. K.S.K., E.R., A.B. and K.H. wrote the manuscript with input from all other co-authors. E.R. rendered the 3D bands and movie.

\section{Competing financial interests}

The authors declare no competing financial interests. 


\section{Figure legends}

Figure 1 I Dirac spectrum of bilayer graphene. a-c, Dirac spectrum of bilayer graphene with $A B, A A$, and twisted AA stacking under electric field $(U=0.4 \mathrm{eV})$. $\mathbf{d}-\mathbf{f}$, Experimental Dirac spectrum of hole-doped (d), near-neutral (e), and electron-doped (f) bilayer graphene, taken at the $\mathrm{K}$ point along $k_{\mathrm{x}}$ (inset in $\mathbf{i}$ ). The photon energy is $95 \mathrm{eV}$ for $\mathbf{d , e}$ and $120 \mathrm{eV}$ for $\mathbf{f}$ (see Supplementary Fig. S2 for the full dataset). Overlaid lines are those from tight-binding band calculations for AB-BG with doping level $n$ (in $10^{13} \mathrm{~cm}^{-2}$ ) and $U$ (in eV) marked at the bottom of each panel. $n$ is quantified from the area of the Fermi surface (assumed circular), and $U$ is estimated from $A B-B G$ bands, as described in ref. 4. $\mathbf{g}-\mathbf{i}$, Corresponding spectral simulations, considering the superposition of tight-binding bands for $A B-B G$ and $A A-B G$ (or tAA-BG), and sublattice interference (Methods). The same simulations only with AB-BG bands are compared in inset. Inset in $\mathbf{i}$ shows the Brillouin zone near the $\mathrm{K}$ point and the measurement directions.

Figure 2 I $n$-doped Dirac spectrum and constant-energy maps. a, Dirac spectrum of highly $n$ doped bilayer graphene taken with $95 \mathrm{eV}$ photons at the $\mathrm{K}$ point along $k_{\mathrm{y}}$ (inset in Fig. 1i). $\mathbf{b}$, Tight-binding bands for AB-BG (black lines) and for AA-BG (red and blue lines, corresponding to $\mathrm{C} 1$ and $\mathrm{C} 2$ in Fig. 1, respectively). Red and blue arrows indicate the pseudospin direction of bands of the same colour. c, Corresponding spectral simulation with the calculated bands in $\mathbf{b}$ (Methods). $\mathbf{d}-\mathbf{h}$, Series of simultaneously acquired k maps at different constant energies as indicated in lower left. Dotted lines are guides for the spectral symmetry. $\mathbf{h}-\mathbf{j}, k$ maps at $E_{\mathrm{D}}$ taken with different photon energies (in $\mathrm{eV}$ ) indicated in upper right. The linear polarization of photons was oriented along $k_{\mathrm{y}}$ for $\mathbf{h}, \mathbf{j}$ and along $k_{\mathrm{x}}$ for $\mathbf{i}$. Red and blue arrows in $\mathbf{h}, \mathbf{i}$ (related to $\mathrm{C} 1$ and $\mathrm{C} 2$, respectively) highlight the reversal of intensity patterns with photon energy. Dashed lines indicated the orientation of the twist-induced symmetry-breaking feature. $\mathbf{k}$, Area enclosed by the circular feature at $E_{\mathrm{D}}$, related to the charge density, as a function of $U$ induced by the controlled Potassium deposition.

Figure 3 I Theoretical band calculations based on the twisted-AA model. a, Tight-binging dispersions for $t A A-B G\left(\theta= \pm 0.15^{\circ}\right.$ and $\left.U=0.4 \mathrm{eV}\right)$. b-g, Simulated constant-energy maps at $E_{\mathrm{D}}$ with $(\mathbf{b}, \mathbf{c})$ and without $(\mathbf{d}-\mathbf{g})$ the interference-related matrix elements (ME). Tight-binding parameters are marked at the bottom of each panel with constant $U=0.4 \mathrm{eV}$ (Methods for details). Dotted lines are guides for rotated spectral symmetry.

Figure $4 \mathrm{I}$ Low-energy band topology and gap structure plotted for $\mathbf{U}=\mathbf{0 . 4} \mathrm{eV}$. a, Threedimensional plot of $t A A-B G$ bands calculated for two representative twist angles. $\mathbf{b}$, Magnified band dispersions near $E_{\mathrm{D}}$ of Fig. $3 a$ for $\theta= \pm 0.15^{\circ} . \Delta$ is a direct gap, and $E_{0}$ is the energy at which the gap is centred. $\mathbf{c}$, Plot of $E_{0}$ and energy extrema of electron and hole bands as a function of azimuthal angle $\phi$ (as defined in $\mathbf{d}$ ) for $\theta= \pm 0.15^{\circ}$. The yellow region enclosed by solid lines corresponds to the energy gap $\Delta$. d, Polar plot of $\Delta(\phi)$ for $\theta= \pm 0.15^{\circ}$. Purple (green) arrows indicate the nodal (antinodal) direction, and the yellow area represents the energy gap $\Delta$. e, Calculated $k$-maps near the $\mathrm{K}$ point at zero energy $\left(E_{\mathrm{D}}\right)$ for two representative twist angles, showing an electronic topological transition. Small arrows represent the pseudospin texture. 

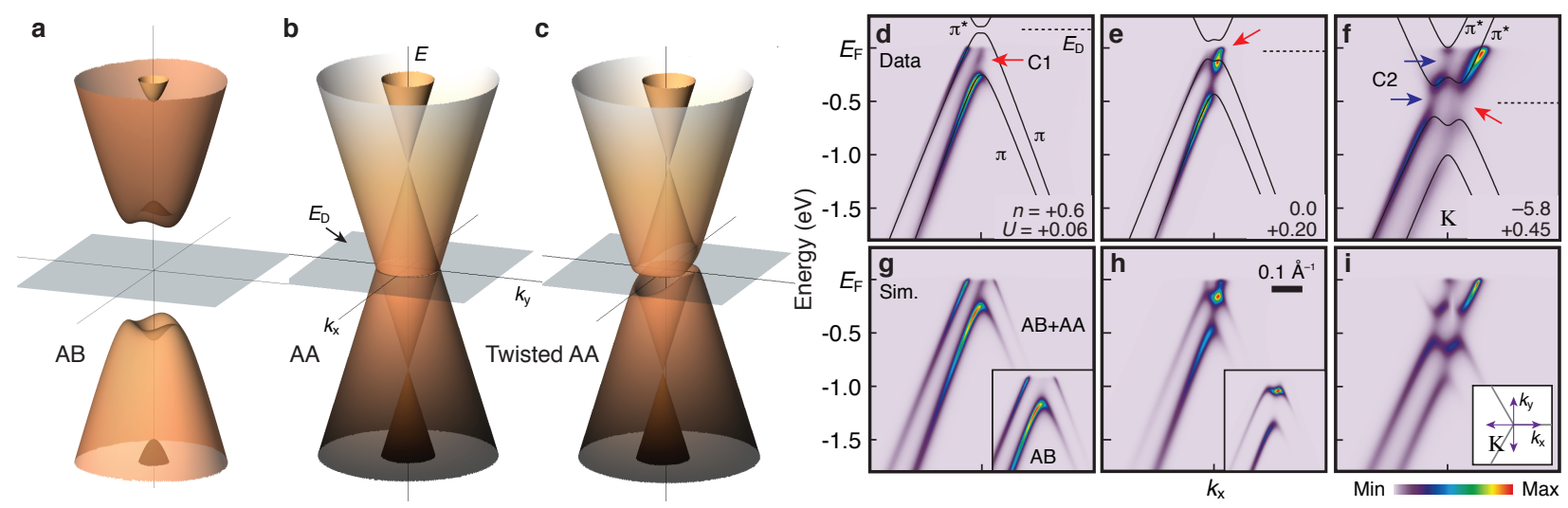


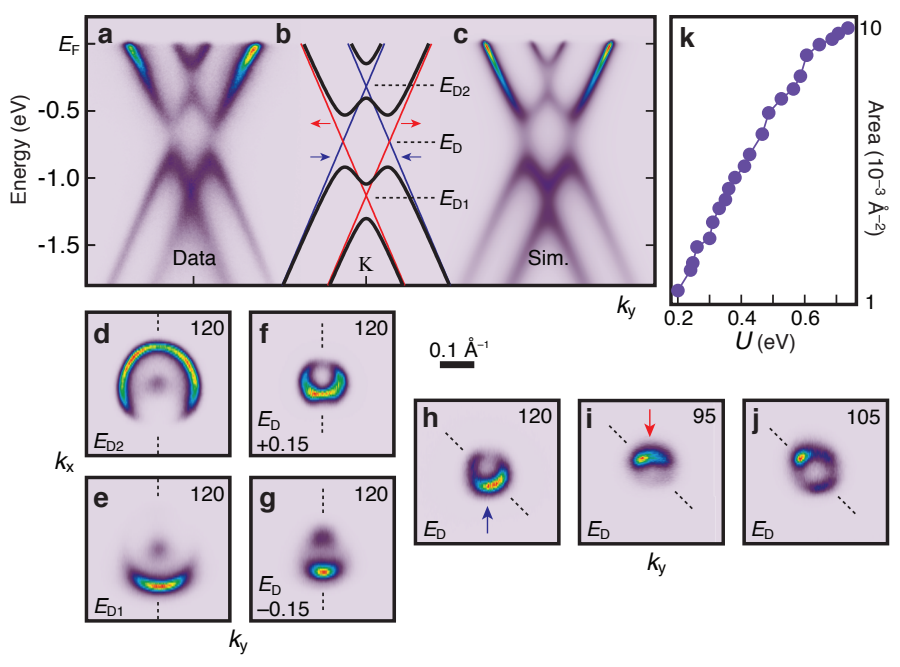




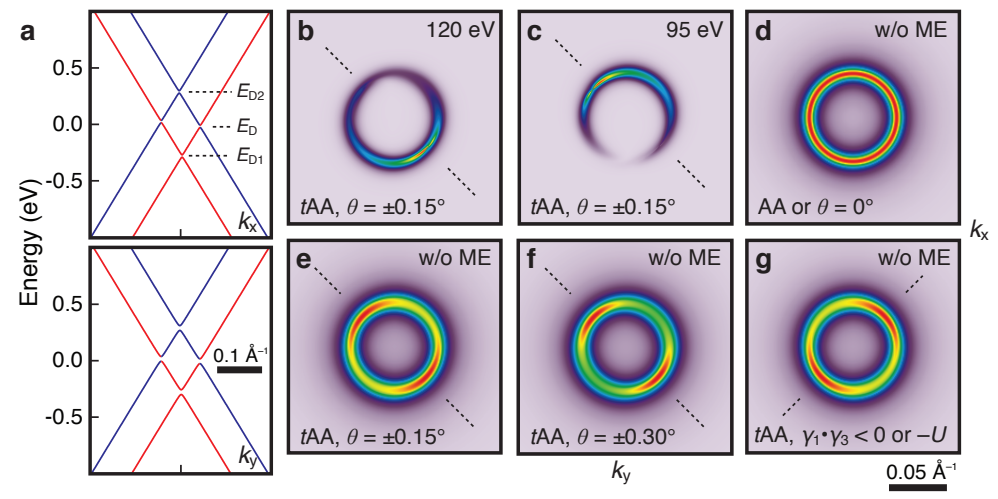


a

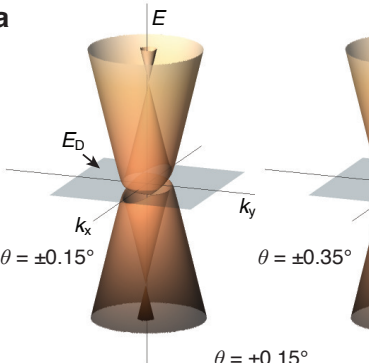

b

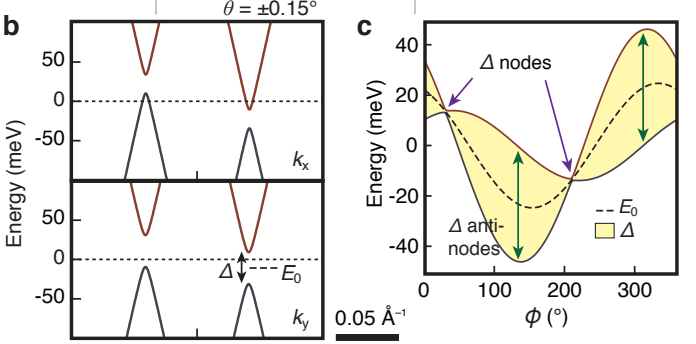

e

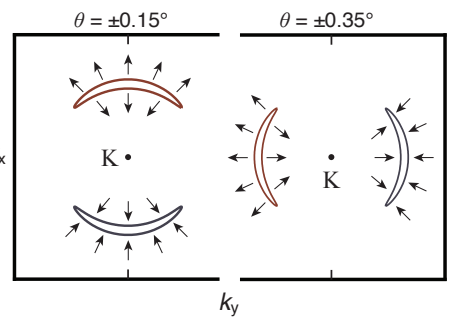

d

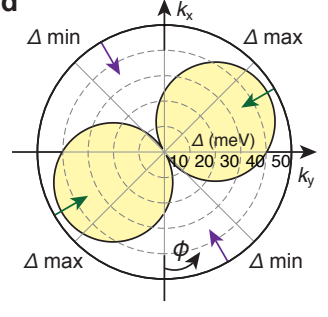




\title{
Coexisting massive and massless Dirac fermions in symmetry-broken bilayer graphene
}

\author{
Keun Su Kim, ${ }^{1,2}$ Andrew L. Walter, ${ }^{3}$ Luca Moreschini, ${ }^{1}$ Thomas \\ Seyller, ${ }^{4}$ Karsten Horn, ${ }^{2}$ Eli Rotenberg, ${ }^{1}$ and Aaron Bostwick ${ }^{1}$ \\ ${ }^{1}$ Advanced Light Source, E. O. Lawrence Berkeley National Laboratory, Berkeley, CA 94720, USA \\ ${ }^{2}$ Department of Molecular Physics, Fritz-Haber-Institut der Max-Planck-Gesellschaft, \\ Faradayweg 4-6, 14195 Berlin, Germany \\ ${ }^{3}$ Donostia International Physics Centre, \\ Manuel Lardizábal 4, E-20018 San Sebastián, Spain \\ ${ }^{4}$ Lehrstuhl für Technische Physik, Universität Erlangen-Nürnberg, \\ Erwin-Rommel-Strasse 1, 91058 Erlangen, Germany
}




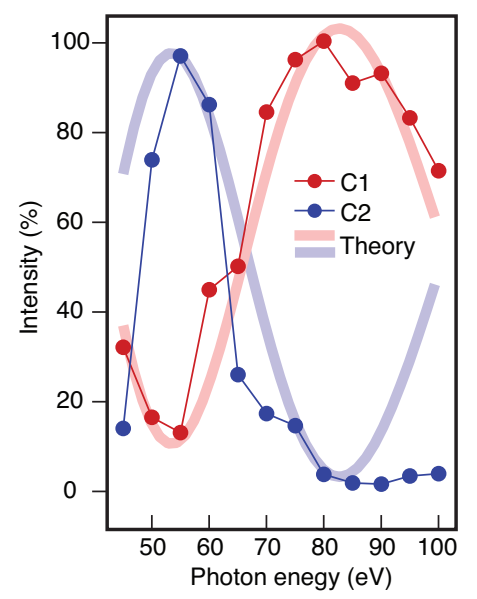

Figure S1 | Interlayer interference of bilayer graphene. Normalized ARPES intensity of C1 and C2 bands in Fig. 1 as a function of photon energy. The data clearly show out-of-phase oscillatory behaviours consistent with theoretical calculations (bold lines) for interlayer-sublattice interference in bilayer graphene (see Methods for details). 


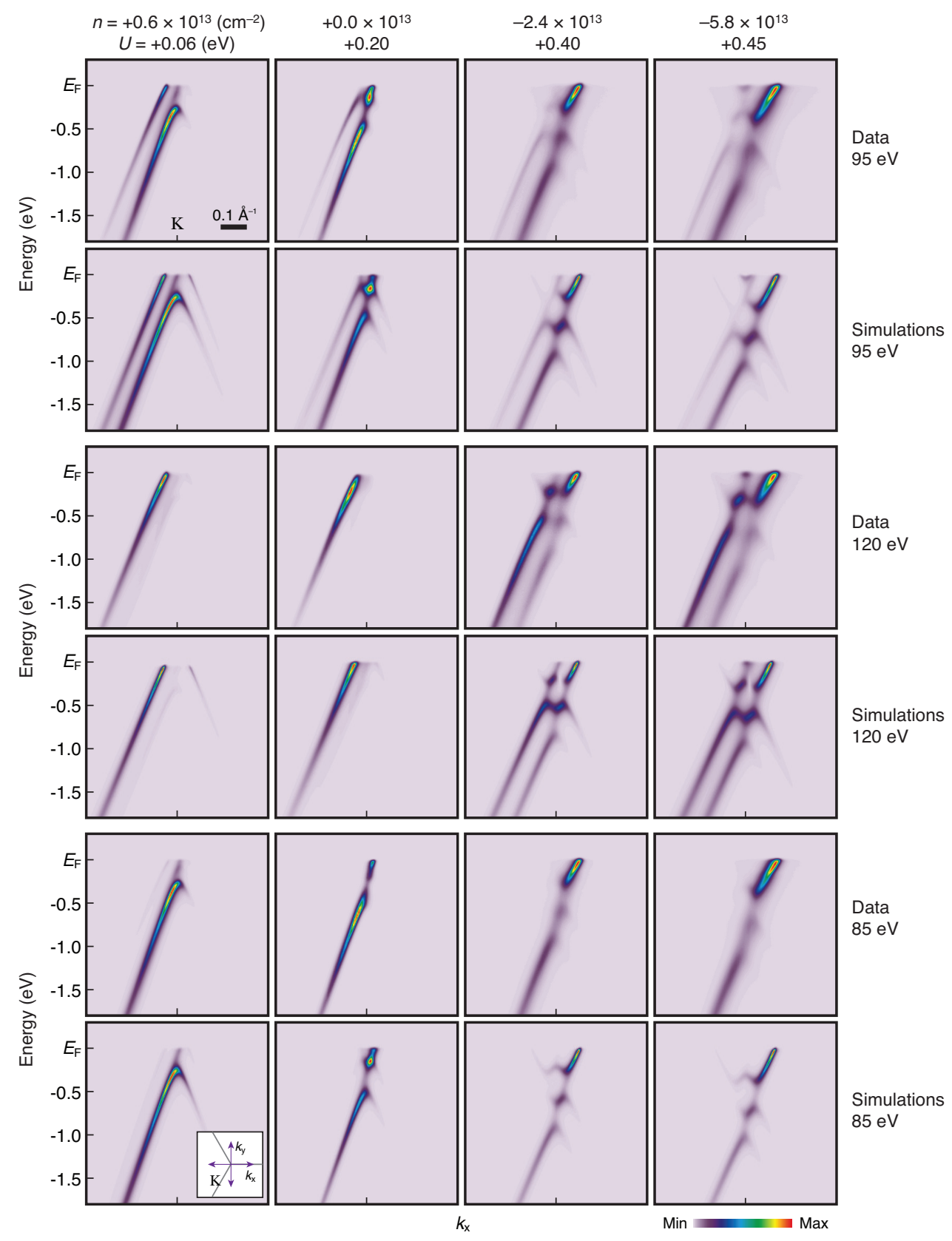

Figure S2 1 Full ARPES dataset and spectral simulations. Doping- and photon-energy set of ARPES spectra, compared with corresponding spectral simulations (Methods). The agreement between data and simulations is remarkable. 


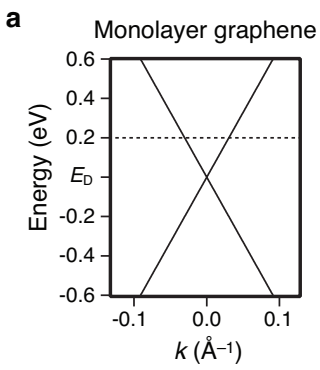

b AA-stacked bilayer graphene
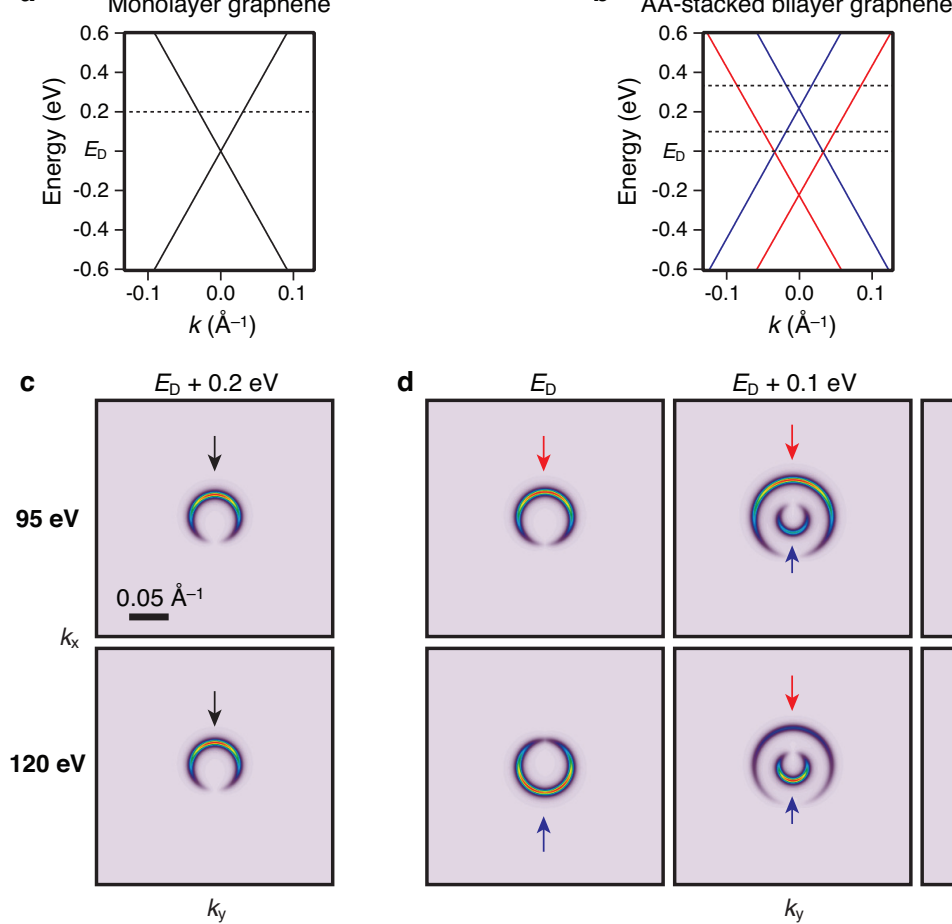

$E_{\mathrm{D}}+0.34 \mathrm{eV}$

Figure S3 | Simulated photon-energy dependence of constant-energy patterns. a,b, Band dispersions of monolayer graphene (a) and AA-BG (b) near $E_{\mathrm{D}}$. Unlike a single Dirac cone in a, double Dirac cones in $\mathbf{b}$ have a special energy $\left(E_{\mathrm{D}}\right)$, at which the two Dirac cones of opposite chirality (red and blue) cross each other to form a doubly degenerated Dirac circle. c,d, Simulated constant-energy maps for monolayer graphene (c) and AA-BG (d) at the energies marked on top of each panel (Methods). The photon energies used to simulate the interference effect are indicated on the left. The simulated maps of a single Dirac cone in $\mathbf{c}$ show a vanishing intensity downwards, independent of photon energy. On the other hand, those of AA-BG at $E_{\mathrm{D}}$ (left panels in d) can be reversed with photon energy, because of the special degeneracy of opposite chirality. Such behaviours do not exist in the maps at other energies (middle and right panels in d). Therefore, the intensity reversal at $E_{\mathrm{D}}$ is a hallmark of the mixed chiralities at $E_{\mathrm{D}}$, expected for AA-BG. 

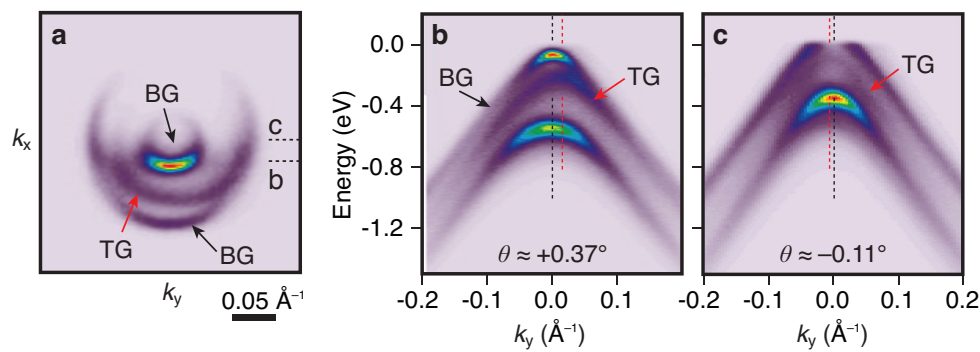

Figure S4 Signature of minute twists. a, Constant-energy map at $E_{\mathrm{D}}-0.6 \mathrm{eV}$, taken from a mixed sample, consisting of separate domains of bilayer (BG) and trilayer (TG) graphene (with the same $n$ and $U$ as Fig. 1d). The band origins were confirmed by the oscillation period of intensity with photon energy (as in Fig. S1). One of three $\pi$ bands of TG is visible with this photon energy $(95 \mathrm{eV})$ due to the interference effect. The TG band is slightly off-centre of BG bands, indicative of a small twist. b,c, Band dispersions along the dotted lines in $\mathbf{a}$, taken at two different spots in a sample. The K points of BG (black dotted line) and TG bands (red dotted line) show small splittings $\Delta k$ with different magnitude and direction. The estimated $\theta$ from $\Delta k$ is about $+0.37^{\circ}$ for $\mathbf{b}$ and $-0.11^{\circ}$ for $\mathbf{c}$, and it varies randomly in between $0.1 \sim 0.4^{\circ}$ over the sample. Even for such a small $\theta, \Delta k=k_{\mathrm{D}} \cdot \theta \cdot \pi / 180$ is still in the measurable range owing to the large $k_{\mathrm{D}} \approx 1.705 \AA^{-1}$ of the Dirac point in graphene. 

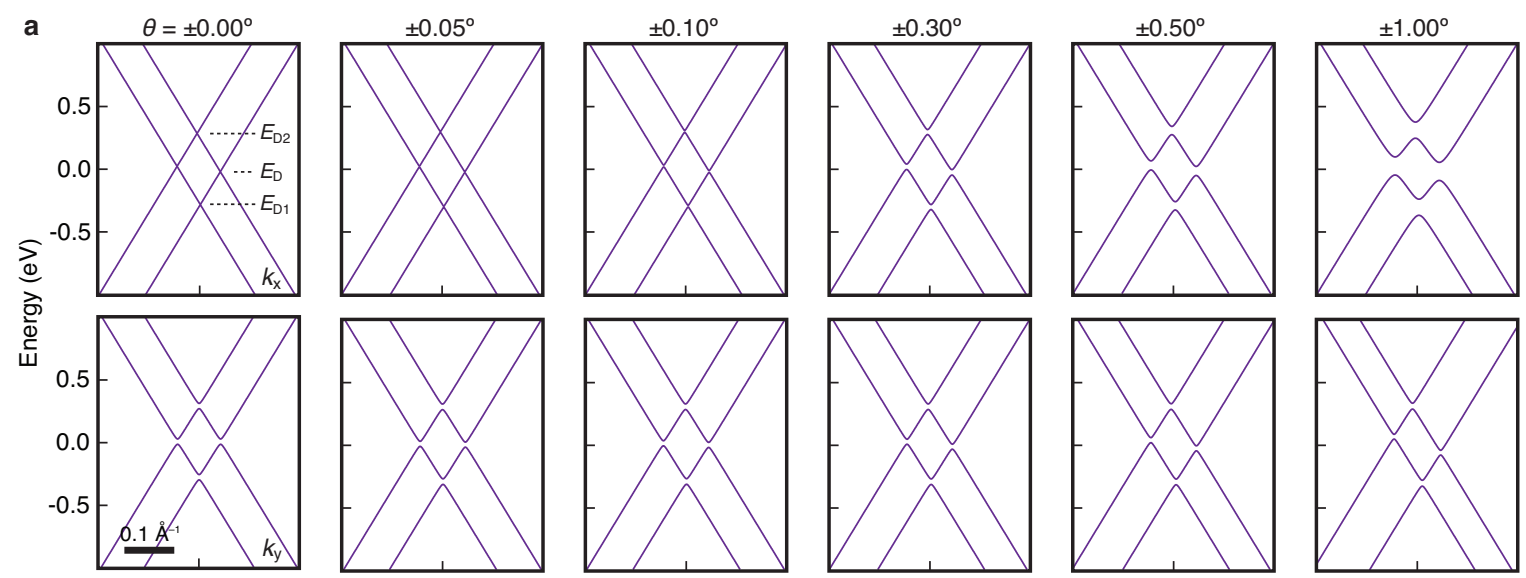

b
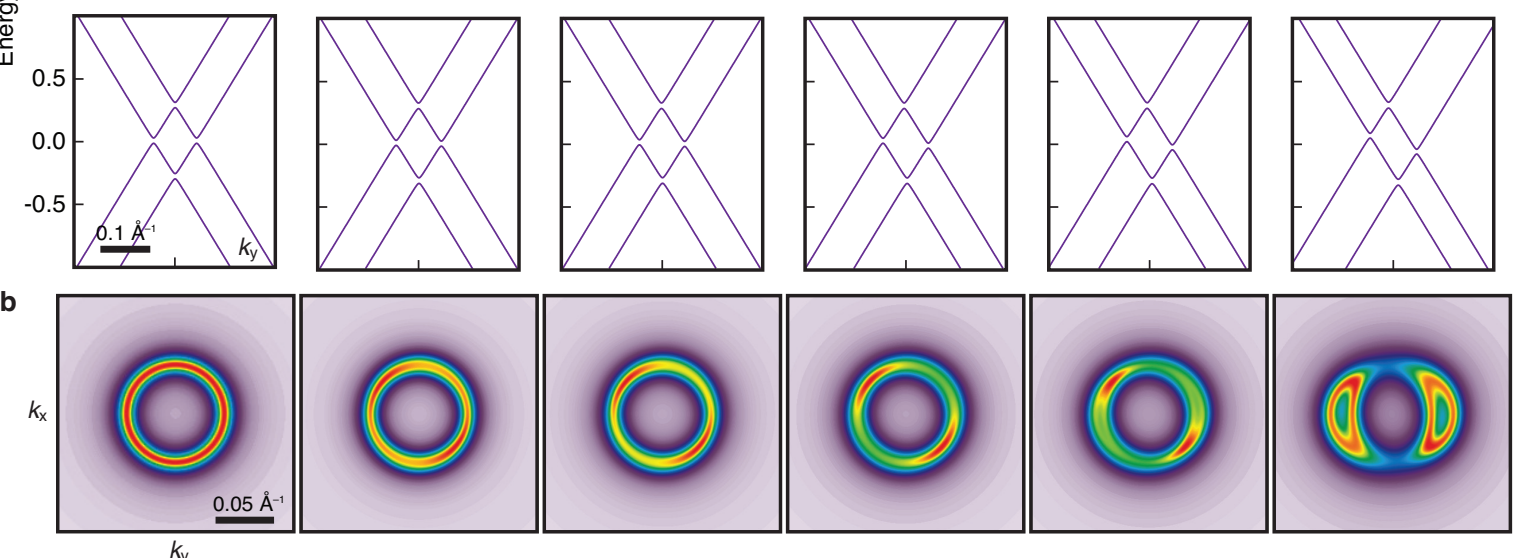

c

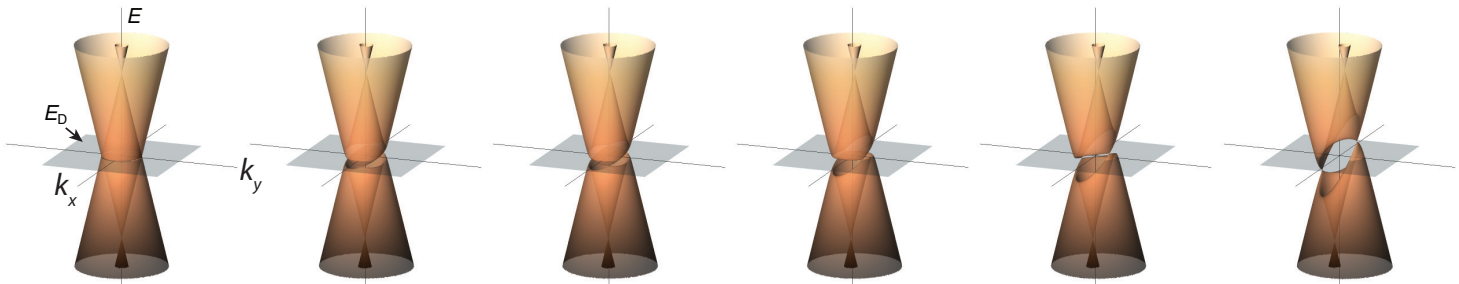

Figure S5 | Full $\theta$ series of theoretical calculations for $\boldsymbol{t A A - B G . ~ a , b , ~ B a n d ~ d i s p e r s i o n s ~ ( a ) ~ a n d ~}$ the spectral-function maps (ignoring matrix element effects) at $E_{\mathrm{D}}$ (b) calculated with different $\theta$ marked at the top. c, Corresponding three-dimensional plots of $t \mathrm{AA}-\mathrm{BG}$ bands near $E_{\mathrm{D}}$. The overall dispersions that resemble untwisted AA-BG and the rotated spectral symmetry at $E_{\mathrm{D}}$ are robust in between $\theta= \pm 0.05 \sim 0.50^{\circ}$, which corresponds to the $\theta$ range observed in Fig. S4. 